\title{
Diagnostic value of standard electrocardiogram in acute right ventricular myocardial infarction
}

\author{
Ali Taherinia (1), Koorosh Ahmadi (1), Mehran Bahramian (1), Peyman
} Khademhosseini (1), Zabihollah Taleshi (1), Mohammadreza Maghsoudi (1), Roya Sattarzadeh Badkoubeh (2), Mohammad Taghi Talebian (3), Mehdi Rezaee (1)

(1) Department of Emergency Medicine, Alborz University of Medical Sciences, Karaj, Iran; (2) Department of Cardiology, Imam Khomeini Hospital, Tehran University of Medical Sciences, Tehran, Iran; (3) Department of Emergency Medicine, Tehran University of Medical Sciences, Tehran, Iran

This article is distributed under the terms of the Creative Commons Attribution Noncommercial License (CC BY-NC 4.0) which permits any noncommercial use, distribution, and reproduction in any medium, provided the original author $(s)$ and source are credited.

\begin{abstract}
Myocardial infarction (MI) (i.e., heart attack) is the irreversible death (necrosis) of heart muscle secondary to prolonged lack of oxygen supply (ischemia) which accounts for a large number of deaths in the hospital. Diagnosis of myocardial infarction is confirmed based on clinical manifestations and electrocardiographic changes along with increased cardiac enzymes. Electrocardiogram (ECG) is one of the safest and easiest methods in the first place. Therefore, this study aimed to investigate the diagnostic value of standard electrocardiogram in the diagnosis of acute right ventricular infarction following lower cardiac infarction. This research was carried out at a time interval of one and a half years to diagnose acute primary infarction. In this method, the diagnostic value of ST $\downarrow$ in lead I, ST $\downarrow$ in lead aVL and I ST $\downarrow+$ aVL, compared with ST $\uparrow$ in lead V4R was investigated for diagnosis of right ventricular infarction. ST $\uparrow$ in the lead V4R is a gold standard for the detection of right ventricular MI. All the patients who had the inclusion criteria were allowed to participate in the study. A total of 66 patients participated in the study. Accordingly, $58(87 \%)$ were male and $8(13 \%)$ were female. The mean age of the population was $54.9 \pm 11.41$. According to the ST $\uparrow$ standard in lead V4R, 26 patients $(39 \%)$ had right ventricular myocardial infarction. There was no significant relationship between angina pectoris and premature infarction $(\mathrm{P}-$ Value $=0.869)$. In this study, the right ventricular was most commonly involved in right coronary artery $(78 \%)$. There was no significant relationship between the occlusion of right coronary artery and right ventricular infarction in 60 patients (PValue $=0.94)$. The results showed that electrocardiogram manifestations help determine the occlusion site and the area at risk (ST $\downarrow$ in lead aVL and aVL + I, sensitivity $=96 \%$ ). In myocardial infarction, symptoms such as the ST-Segment elevation in lead aVR and ST-Segment depression in the lower leads are possible. Accordingly, in the lower infarction, ST changes in the leads V1-V6 are helpful in detecting patients at risk. Thus, the use of electrocardiogram in acute myocardial infarction helps detect more invasive patients and prevents extensive myocardial damage and other complications.
\end{abstract}

Key Words: Electrocardiogram, myocardial infarction, right ventricle

Eur J Transl Myol 29 (2): 130-135, 2019

Myocardial infarction (MI) is the irreversible death (necrosis) of heart muscle secondary to prolonged lack of oxygen supply (ischemia) which accounts for a large number of deaths in the hospital. ${ }^{1}$ Some of the main risk factors of MI include diabetes, hypertension, hyperlipidemia, tobacco, alcohol, obesity, family history, and gender. Given the increasing prevalence of the disease in developed and developing countries, the average age of patients suffering from MI is decreasing. ${ }^{2}$ In Iran, heart disease accounts for about $46 \%$ of all deaths. ${ }^{3}$ Diagnosis of MI is confirmed based on clinical manifestations and electrocardiographic changes along with increased cardiac enzymes in the blood. An electrocardiogram (ECG) is a medical test that detects heart problems by measuring the electrical activity generated by the heart as it contracts. ${ }^{4}$ 
Over the past two decades, several studies have investigated the involvement of myocardial infarction with right ventricle (RV) and its accompaniment with more complications and increased mortality in these patients. ${ }^{5}$ According to studies, 18 to $84 \%$ of the cases of MI are associated with right ventricular myocardial infarction (RVMI). ${ }^{6}$ The incidence of RVMI complicates the prognosis. The mortality rate of RVMI and $\mathrm{MI}$ is 25\%-30\%, while MI accounts for 6\%..$^{7}$ Patients with inferior MI who also have RV myocardial involvement are at increased risk of death, shock and arrhythmias. This increased risk is related to the presence of $\mathrm{RV}$ myocardial involvement itself rather than the extent of left ventricular (LV) myocardial damage. Ischemia and RVMI-related right ventricular dysfunction can be treated upon timely diagnosis. This illustrates the importance of quick and timely diagnosis of RVMI. Accurate and timely diagnosis of these patients is essential in order to improve their final outcome. The American Heart Association introduces ECG as a useful and accessible tool for the diagnosis of right ventricular MI, which reflects RVMI-related changes. Abnormalities detected by ECG help doctors determine the type of treatment needed. The abnormalities on ECG also help show where the heart muscle was damaged. If a person has had previous heart problems, which can alter the ECG, the most recent damage may be harder for doctors to detect. This tool can also be used for clinical examinations, laboratory tests and those with suspected acute heart disease. ${ }^{8}$ Using ECG, patients with myocardial infarction and suspected of having acute heart problems are provided with valuable information on the initial assessment and severity of risk. ${ }^{9}$ If ECG changes in favor of reducing heart rate, usually a person is admitted to the coronary care unit (CCU). Otherwise, the doctor will decide, depending on the examinations and patient history. ECG has its own limitations and should not be used as the sole means of diagnosing heart disease because many heart diseases cannot be detected by the ECG. ${ }^{10}$ Typically, about $50 \%$ of patients with unstable angina (UA) and non-ST elevation myocardial infarction (NSTEMI) do not show any changes in the ECG in the first place. UA and the closely related condition of NSTEMI are very common manifestations of this disease. Therefore, it is necessary to obtain a precise history of the patient. Likewise, the correct selection of each diagnostic device for every patient is of utmost importance. ${ }^{11}$ Therefore, this study aimed to evaluate the diagnostic value of electrocardiogram in the diagnosis of acute right ventricular infarction.

\section{Materials and Methods}

This research was carried out at a time interval of one and a half years to diagnose acute primary myocardial infarction., which was carried out at the Tehran Heart Center. In this method, the ST elevation ST value $\downarrow$ was tested on lead I, ST $\downarrow$ in the aVL lead and ST $\downarrow$ in the I + aVL lead compared to ST $\uparrow$ in the V4R lead for right

\section{Table 1. Inclusion and exclusion criteria}

\section{Inclusion criteria}

No previous history of myocardial infarction

The onset of symptoms less than 6 hours

Sinusoidal rhythm in electrocardiogram

No Bundle Blok and ST $\uparrow$ (more than $1 \mathrm{~mm}$ ) in the adjacent leads II, III, avf

\section{Exclusion criteria}

Incomplete case

A previous history of myocardial infarction

ventricular MI diagnosis. ST $\uparrow$ in the lead V4R is a gold standard for the detection of right ventricular MI. The sample included all the patients who were allowed to participate in the study. Inclusion and exclusion criteria are listed in Table 1. The sample size included 73 patients who participated in the study with the initial diagnosis of myocardial infarction. Accordingly, 7 patients were excluded. In this study, all patients who were suspected of acute myocardial infarction underwent an electrocardiogram. In the case of ST $\uparrow$ (more than $1 \mathrm{~mm}$ ) in the adjacent II, III avf, patients underwent additional electrocardiograms. The enzyme changes were recorded in order to confirm the diagnosis. According to the AHA/ACC Guidelines of the American College of Cardiology, coronary angiography was performed. After collecting the indexes, the electrocardiograms were individually read by the cardiologist and the emergency medicine specialist and the changes were recorded in encoded forms. The ST was measured at $60 \mathrm{msec}$ from the $\mathrm{J}$ point and values less than $0.5 \mathrm{~mm}$ were assumed as isoelectric. Then, according to the standard criteria (ST $\uparrow$ in lead V4R), patients were divided in two groups with right ventricular involvement and without right ventricular involvement. Finally, diagnostic tests (ST $\downarrow$ in lead I, ST $\downarrow$ in lead aVL and ST $\downarrow$ in lead I + aVL) were investigated in two groups and compared with the original ST $\uparrow$ standard in lead V4R. The patients were examined for angina pectoris before infarction once during the admission by a specialist and again during the first 2 days of admission to the CCU by a cardiologist. During the admission, the patients underwent angiography according to the American Heart Association (AHA), and the degree and occlusion site of coronary artery were determined. The findings were compared with the initial diagnosis of right ventricular infarction (based on chest pain for more 30 minutes and enzyme changes and ST $\uparrow$ in lead V4R in more than 1 $\mathrm{mm}$ ). Finally, sensitivity, specificity, positive predictive value and negative predictive value were calculated.

\section{Ethical Ethical considerations}

This study was approved by the ethics committee of Tehran University of Medical Sciences (code \# IRTC.1386.12.12). In this study, no intervention was made in the diagnosis and admission of patients. 
Table 2. Relationship between angina pectoris before infarction and right ventricular involvement

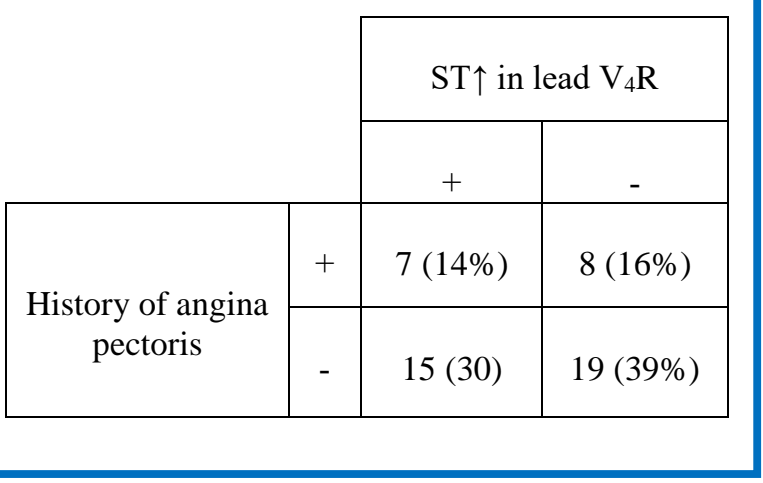

\section{Results}

66 patients participated in the study. 58 patients $(87 \%)$ were male and $8(13 \%)$ were female. The participants were $54.9 \pm 11.41$ years old on average. According to the ST $\uparrow$ standard, in lead $\mathrm{V}_{4} \mathrm{R}, 26$ patients (39\%) had right ventricular myocardial infarction. Likewise, 40 patients (61\%) had problems only in the heart muscle. Of 26 patients with right ventricular involvement, 24 patients had ST $\downarrow$ in lead I, $(1 \mathrm{~mm}<)$. Moreover, of 40 patients without right ventricular involvement, 31 patients did not show ST $\downarrow$ in lead I $(1 \mathrm{~mm} \leq)$. According to the ST $\uparrow$ standard, the following results were obtained: ST $\downarrow$ in lead I $(1 \mathrm{~mm} \leq)$, sensitivity $=92.3 \%$, specificity $=77.5 \%$, positive predictive value $=72.73 \%$, negative predictive value $=93.94 \%$, positive likelihood ratio $=4$ and negative likelihood ratio $=0.1$. Of 26 patients with right ventricular involvement, 25 patients had ST $\downarrow$ in lead avl $(\mathrm{mm} \leq 1)$. Of 40 patients without right ventricular involvement, 18 patients did not show ST $\downarrow$ in lead avl. Finally, based on V4R, the following results were obtained: ST $\downarrow$ in lead avl $(1 \mathrm{~mm} \leq)$, sensitivity $=96.15 \%$, specificity $=45 \%$, positive predictive value $=53.19 \%$, negative predictive value $=94.74 \%$, positive likelihood ratio $=1.7$ and negative likelihood ratio $=0.08$. Finally, of 26 patients with right ventricular involvement, 25
Table 3. Relationship between occlusion of proximal $R C A$ and right ventricular $M I$

\begin{tabular}{|c|c|c|c|}
\hline & & \multicolumn{2}{|c|}{$\mathrm{ST} \uparrow$ in lead $\mathrm{V}_{4} \mathrm{R}$} \\
\hline & & + & - \\
\hline \multirow{2}{*}{$\begin{array}{l}\text { Proximal right } \\
\text { coronary artery } \\
\text { involvement }\end{array}$} & + & $19(32 \%)$ & $21(35 \%)$ \\
\hline & - & $5(8 \%)$ & $15(25 \%)$ \\
\hline
\end{tabular}

patients had ST $\downarrow$ in lead aVL + I $(2 \mathrm{~mm} \leq)$. Likewise, of 40 patients without right ventricular involvement, 33 patients did not show ST $\downarrow$ in lead aVL + I $(2 \mathrm{~mm} \leq)$. Finally, based on the ST $\uparrow$ criterion, the following results were obtained: ST $\downarrow$ in I + avL $(2 \mathrm{~mm} \leq)$, sensitivity $=$ $96.15 \%$, specificity $=82.5 \%$, positive predictive value $=$ $78.13 \%$, negative predictive value $=96.06 \%$, positive likelihood ratio $=5.4$ and negative likelihood ratio $=$ 0.048. Table 2 investigates the relationship between angina pectoris and right ventricular involvement in 49 patients with myocardial infarction in Tehran Heart Center $(\mathrm{P}$-value $=0.869)$. There was no significant relationship between the absence of chest pain 72 hours prior to acute myocardial infarction (AMI) and right ventricular infarction, indicating that chest pain in all cases was not associated with acute myocardial infarction and right ventricular infarction. Table 3 investigates the relationship between the occlusion of proximal right coronary artery (RCA) and right ventricular myocardial infarction in 60 patients referring to the Tehran Heart Center. Although the most common case of right ventricular involvement following obstruction was proximal RCA $(78 \%)$ (P-value $=0.94)$, there is no significant relationship between right ventricular involvement and proximal RCA involvement. Table 4 investigates the relationship between the ratio of ST $\downarrow$ in

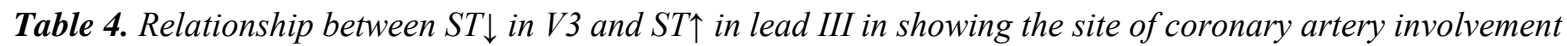

\begin{tabular}{|c|c|c|c|c|c|}
\hline \multicolumn{5}{|c|}{ III ST $\uparrow / V 3$ ST $\downarrow$} & \multirow{2}{*}{ Total } \\
\hline & & $<0.5$ & $0.5-1.2$ & $>1.2$ & \\
\hline & $\begin{array}{ll}\text { Proximal right } \\
\text { coronary artery }\end{array}$ & 29 & 4 & 7 & 40 \\
\hline \multirow{2}{*}{ Angiography } & Right distal coronary & 6 & 1 & 1 & 8 \\
\hline & Circumflex & 6 & 2 & 4 & 12 \\
\hline \multicolumn{2}{|c|}{ Total } & 41 & 7 & 12 & 60 \\
\hline
\end{tabular}




\section{ECG in acute right ventricular myocardial infarction}

Eur J Transl Myol 29 (2): 130-135, 2019

lead V3 and ST $\uparrow$ in Lead III in 60 patients diagnosed with acute myocardial infarction. There was no significant relationship between the ST $\downarrow$ in lead $\mathrm{V} 3$ and $\mathrm{ST} \uparrow$ in lead III (P-value $=0.60)$; particularly in the occluded coronary artery: peripheral, distal and circumflex. ${ }^{12}$ Therefore, proximal RCA, right distal coronary artery and circumflex artery are not involved. Table 4 investigates the relationship between the ratio of ST $\downarrow$ in lead V3 and $\mathrm{ST} \uparrow$ in Lead III in 60 patients diagnosed with acute myocardial infarction. There was no significant relationship between the ST $\downarrow$ in lead V3 and ST $\uparrow$ in lead III (P-value $=0.60)$; particularly in the occluded coronary artery: peripheral, distal and circumflex. ${ }^{12}$ Therefore, proximal RCA, right distal coronary artery and circumflex artery are not involved.

\section{Discussion}

Myocardial infarction often occurs as a result of sudden coronary artery failure, causing necrosis and inappropriate cardiac muscle function. ${ }^{13}$ Treatment is often a percutaneous coronary intervention, ${ }^{14}$ but an early and precise ECG diagnosis is mandatory. RVMI indicates damage to the right ventricular muscle. It should be noted that right ventricular involvement is rare. The 12-lead ECG paints a picture of the left ventricle, while the right ventricle can be evaluated to a very small extent in the standard ECG. ${ }^{15}$ The standard 12-lead electrocardiogram is one of the most commonly used medical studies in the assessment of cardiovascular disease. It is the most important test for interpretation of the cardiac rhythm, detection of myocardial ischemia and infarction, conduction system abnormalities, preexcitation, long QT syndromes, atrial abnormalities, ventricular hypertrophy, pericarditis, and other conditions. Therefore, ECG was used to diagnose acute right ventricular myocardial infarction. A total of 66 patients (58 males and 8 females) participated in the study. The incidence of right ventricular myocardial infarction was 39\%. Although acute right ventricular MI can occur due to the involvement of any main coronary artery, it is often attributed to right coronary artery. In this study, 22 males and 4 females suffered from right ventricular MI involvement, which was similar to that of Aygul, Hosseini et al. ${ }^{16,17}$ Turhan et al. concluded that ST $\downarrow$ in aVL was greater than $1 \mathrm{~mm}$ with a sensitivity of $87 \%$ and a specificity of $91 \%$. Rashduni et al. observed in 22 patients with acute myocardial infarction that ST $\downarrow$ in aVL $(1 \mathrm{~mm} \leq)$ had a sensitivity of $100 \%$ and a specificity of $57 \%$. In this study, ST $\downarrow$ in $\operatorname{aVL}(1 \mathrm{~mm} \leq)$ had a sensitivity of $96 \%$, a specificity of $45 \%$, a positive predictive value of $53 \%$ and a negative predictive value of $94.5 \%$ (based on the $\mathrm{ST} \uparrow$ in $\mathrm{V}_{4} \mathrm{R}$ ). However, Turhan et al. showed a higher sensitivity and a lower specificity. It can be attributed to the fact that we included $1 \mathrm{~mm} \leq$ in this study, but they considered more than $1 \mathrm{~mm} .{ }^{18,19}$ In this study, the $\mathrm{ST} \downarrow$ in lead $\mathrm{I}(1 \mathrm{~mm} \leq)$ had a sensitivity of $92 \%$, a specificity of $77 \%$, a positive predictive value of $72 \%$, and a negative predictive value of $93 \%$. Likewise, lead I may be the only lead in the standard ECG which reflects the changes in the right ventricle. Although many attempts have been made to diagnose RVMI based on ST changes in the 12-lead ECG, few studies have focused on 12-lead ECG. ${ }^{20,21}$ However, in all patients with myocardial infarction, ST changes in lead I should be considered. Mittal et al. examined 24 patients and concluded that ST $\downarrow$ in lead I and aVL has a high sensitivity in the diagnosis of right ventricular MI, which is in good agreement with this study. ${ }^{22} \mathrm{Pu} \mathrm{SY}$, et al. examined 50 patients with acute right ventricular myocardial infarction and concluded that aVL+ I had a sensitivity of $96 \%$ and a specificity of $89 \%$ in the diagnosis of acute right ventricular myocardial infarction. This research aimed to increase the sensitivity and negative predictive value. The results showed that $\mathrm{aVL}+\mathrm{I}(2 \mathrm{~mm} \leq)$ had a sensitivity of $96.15 \%$, a specificity of $82 \%$, a positive predictive value of $78 \%$ and a negative predictive value of $97.06 \%$. The sample size of this study was about $30 \%$ greater than that of Pu SY et al. (66 versus 50). The specificity of this study was lower than that of $\mathrm{Pu}$ SY et al., which could be attributed to the fact that this study aimed to achieve a higher sensitivity and higher negative predictive value. ${ }^{23}$ The relationship between proximal RCA involvement and right ventricular myocardial infarction was also investigated, despite that fact the most common cause of right ventricular involvement was the occlusion of right coronary artery (78\%). Although isolated right ventricular MI is usually seen in patients suffering from chronic lung disease together with right ventricular hypertrophy, it can occur in patients suffering a transmural infarction of the inferior-posterior wall which extends to involve the right ventricular wall as well. Right ventricular MI is most commonly caused by obstruction of the proximal right coronary artery and is frequently associated with right bundle branch block. Furthermore, only 5\% - 10\% of patients suffer from hemodynamic symptoms. However, right ventricular myocardial infarction occurred in $22 \%$ of cases as a result of the occlusion of other arteries. This result was in good agreement with that of William et al. Of 40 patients with the occlusion of proximal RCA, only 19 cases resulted in right ventricular myocardial infarction. It should be noted that right ventricular myocardial infarction occurs commonly as a result of pulmonary hypertension. Therefore, the occlusion of proximal RCA does not necessarily cause right ventricular myocardial infarction. ${ }^{24}$ The present study also aimed to investigate the relationship between angina pectoris and right ventricular myocardial infarction. This was not in good agreement with Shiraki et al. It is not possible to obtain a precise and reliable biographical report, given the special emergency conditions. Thus, it is not possible to comment on this with certainty. ${ }^{25}$ The results showed that electrocardiogram manifestations help to determine the site of the obstruction and the area at risk (ST $\downarrow$ in aVL and aVL + I with a sensitivity of $96 \%$ ). Myocardial infarction is associated with 
symptoms such as ST-Segment elevation in aVR and STSegment depression in the lower leads. ST changes in V1-V6 may help detect patients at risk. Therefore, otherwise unexplained systemic arterial hypotension or diminished cardiac output, or marked hypotension in response to small doses of nitroglycerin in patients with inferior infarction, should lead to the prompt consideration of this diagnosis. Successful reperfusion of the right coronary artery significantly improves right ventricular mechanical function and lowers in-hospital mortality in patients with right ventricular infarction. In conclusion, ECG helps to detect the more critical patients with acute myocardial infarction and to prevent extensive myocardial damage and other complications..

\section{List of acronyms}

AMI - acute myocardial infarction

$\mathrm{CCU}$ - coronary care unit

ECG - electrocardiogram

LV - left ventricul

MI - myocardial infarction

NSTEMI - non-ST-segment elevation MI

RCA - right coronary artery

$\mathrm{RV}$ - right ventricular

UA - unstable angina

\section{Authors contributions}

All authors played a substantial role in the conception and/or study design, data acquisition and/or analysis, as well as drafting of the manuscript, approval of the final version to be published.

\section{Acknowledgments None. Funding None}

\section{Conflict of Interest}

The authors declare they have no conflicts of interest.

\section{Ethical Publication Statement}

We confirm that we have read the Journal's position on issues involved in ethical publication and affirm that this report is consistent with those guidelines.

\section{Corresponding Author}

Mehdi Rezaee, Department of Emergency Medicine, Alborz University of Medical Sciences, Karaj, Iran. Phone: 00989124047348

Email: drmehdirezaeemd@yahoo.com

E-mails of co-authors

Ali Taherinia: dr.alitaheriniya@gmail.com

Koorosh Ahmadi: kooroshem@gmail.com

Mehran Bahramian:dr_mehran_b@yahoo.com

Peyman Khademhosseini: Peyman_kh53@yahoo.com

Zabihollah Taleshi: zabitaleshi@yahoo.com

Mohammadreza Maghsoudi:

dr.maghsoudi.m@gmail.com

Roya sattarzadeh badkoubeh: satarzad@tums.ac.ir

Mohammad Taghi Talebian:

mohammadtaghitalebian@gmail.com

\section{References}

1. Manfroi WC, Peukert C, Berti CB, et al. Acute myocardial infarction: the first manifestation of ischemic heart disease and relation to risk factors. Arq Bras Cardiol 2002;78:392-5.

2. Mcaig LF, Nawar EW. National Hospital Ambulatory Medical Care Survey: 2004 emergency department summary. Adv Data 2006;37:1-29.

3. Naghavi M. The picture of death in 18 provinces of Iran in 2001, Iran. Ministry of Health and Medical Educations Publications 2003;14:12-3.

4. Arnin J, Yamsa-Ard T, Triponyuwasin P, Wongsawat Y. Development of practical functional electrical stimulation cycling systems based on an electromyography study of the Cybathlon 2016. Eur J Transl Myol 2017 Dec 5;27(4):7111. doi: 10.4081/ejtm.2017.7111. eCollection 2017 Dec 5.

5. Hamon M, Agostini D, Le Page O, Riddell JW. Prognostic impact of right ventricular involvement in patients with acute myocardial infarction: metaanalysis. Crit Care Med 2008;3:2023-33.

6. Carter T, Ellis K. Right-ventricular infarction. Crit Care Nurse 2005;2:52-60.

7. Navarro C, Owens C, Riddell J, et al. The use of calculated epicardial potentials improves significantly the sensitivity of a diagnostic algorithm in the detection of acute myocardial infarction. J Electrocardiol 2003;3:127-32.

8. Ting HH, Krumholz HM, Bradley EH, et al. Implementation and integration of prehospital ECGs into systems of care for acute coronary syndrome: a scientific statement from the American Heart Association Interdisciplinary Council on Quality of Care and Outcomes Research, Emergency Cardiovascular Care Committee, Council on Cardiovascular Nursing, and Council on Clinical Cardiology. Circulation 2008;118:106679.

9. Shahmirzaee R, Sadrnia S, Solhi H, Ghasemi A. The evaluation of electrocardiogram in patients with unstable angina and non-ST elevation myocardial infarction. Arak Medical University Journal (AMUJ) 2010;13:83-90.

10. Swap C, Nagurney J. Value and limitations of chest pain history in the evaluation of patients with suspected acute coronary syndromes. Jama 2005;294:2623. Erratum in JAMA 2006;295:2250.

11. Tong K, Kaul S, Wang X, et al. Myocardial contrast echocardiography versus Thrombolysis in Myocardial Infarction score in patients presenting to the emergency department with chest pain and a nondiagnostic electrocardiogram. J Am Coll Cardiol 2005;46:920-7.

12. Kosuge $\mathrm{M}$, Kimura $\mathrm{K}$, Ishikawa $\mathrm{T}$, et al. Implication of the absence of st-segment elevation in lead V4R in patients who have inferior wall acute myocardial infarction whit right ventricular involvement. Clin cardiol 2001;24:225-30. 


\section{ECG in acute right ventricular myocardial infarction}

Eur J Transl Myol 29 (2): 130-135, 2019

13. Ginghina C, Caloianu GA, Serban M, Dragomir D. Right ventricular myocardial infarction and pulmonary embolism differential diagnosis - a challenge for the clinician. J Med Life 2010;3:24253.

14. Sharifi Z, Yazdi MJ, Eshraghi A, et al. Clinical outcomes and complications of treatment with supraflex stent in patients with coronary artery disease: One year follow up. Eur J Transl Myol 2019;29(2):8231. doi: 10.4081/ejtm.2019.8231.

15. Moye S, Carney MF, Holstege C, et al. The electrocardiogram in right ventricular myocardial infarction. Am J Emerg Med 2005;2:793-9.

16. Aygul N, Ozdemir K, Abaci A, et al. Prevalence of risk factors of ST segment elevation myocardial infarction in Turkish patients living in Central Anatolia. Anadolu Kardiyol Derg 2009;9:3-8.

17. Hosseini SK, Soleimani A, Salarifar M, et al. Demographics and Angiographic Findings in Patients under 35 Years of Age with Acute ST Elevation Myocardial Infarction. J Tehran Heart Cent 2011;6:62-7.

18. Rashduni DL, Tannenbaum. Utility of ST segment depression in lead AVL in diagnosis of right ventricular infarction. N J Med 2003;100:35-3.

19. Turhan H, Yilmaz MB, Yectin E, et al. Diagnostic value of avl derivation for right ventricular involvement in patients with acute myocardial infarction. Ann Noninvasive Electrocardiol $2003 ; 12: 185-8$
20. Tsuka Y, Sugiura T, Hatada K, et al. Clinical significance of ST-segment elevation in lead V1 in patients with acute inferior wall Q-wave myocardial infarction. Am Heart J 2001;14:615-20.

21. Saw J, Davies C, Fung A, et al. Value of ST elevation in lead III greater than lead II in inferior wall acute myocardial infarction for predicting inhospital mortality and diagnosing right ventricular infarction. Am J Cardiol 2001;8:448-50.

22. Mittal SR, Jain S. Electrocardiographic diagnosis of right ventricular infarction in presence of left ventricular posterior infarction. Int $\mathrm{j}$ cardiol 1999;68:125-8.

23. Pu SY, Iuang JL, Chen CK, et al. Prediction of right ventricular infarction from standard surface ECG in patient with inferior myocardial infarction. Zhonghua Yi Xue Za Zhi (Taipei) 1998;61:253-9.

24. Brady WJ, Harrigan RA, Chan T. Acute Coronary Syndromes. In: Marx J, Walls R, Hockberger R. Rosen's emergency medicine: concepts and clinical practice. 6nd ed. Philadelphia: Mosby 2006;9:115498.

25. Shiraki H, Yoshikawa T, Anzai T, et al. Association between preinfarct angina and a lower risk of right ventricular infarction. N Engl J Med 1998;338:9417.

Submissions: $\quad$ March, 24, 2019

Revision received: April 12, 2019

Acceptance: April 15, 2019 